\title{
A percepção de acadêmicos de odontologia sobre o PET-Saúde UFMS/ SESAU, Campo Grande/MS, 2009
}

\author{
Milca Lopes de Oliveira*, Tenile Carvalho Coelho** \\ * Doutora em Saúde Pública, tutora do PET-Saúde UFMS/SESAU \\ 2009-2011 \\ ** Cirurgiã-dentista. Acadêmica PET-Saúde UFMS / SESAU 2009-2010
}

\section{RESUMO}

Introdução: O Programa de Educação pelo Trabalho para a Saúde - PET-Saúde, deve estimular a formação de profissionais e docentes de elevada qualificação técnica, científica, tecnológica e acadêmica, bem como a atuação profissional pautada pelo espírito crítico, pela cidadania e pela função social da educação superior, orientados pelo princípio da indissociabilidade entre ensino, pesquisa e extensão. Com o Convênio da Universidade Federal de Mato Grosso do Sul (UFMS) com a Secretaria Municipal de Saúde (SESAU), criou-se o Programa com sete cursos no ano de 2009, em duas linhas de pesquisa. Objetivos: relatar a percepção de acadêmicos do curso de Odontologia da UFMS sobre o PET-Saúde 2009 e seu impacto na formação acadêmica. Material e Métodos: Relato de experiência tendo como fontes de dados relatórios mensais de acadêmicos de Odontologia do PET-Saúde 2009 e documentos de acompanhamento dos mesmos. Resultados: Dos 60 acadêmicos de sete cursos da área da Saúde, cinco cursavam Odontologia e desses, três eram bolsistas. Constata-se um impacto relevante na formação acadêmica, pois os aspectos multiprofissionalidade, integração teoria e prática, produção de conhecimentos na área da Saúde Coletiva, princípios do SUS, funcionamento de uma UBSF, capacidade de diálogo, respeito aos colegas e tomada de decisões, ficaram evidentes. Acredita-se que a satisfação em ser petiano tenha sido um elemento motivador e de sensibilização para a atuação e o aprendizado. Conclusões: Espera-se que Instituições de Ensino Superior e Secretarias de Saúde dos Municípios sejam bastante permeáveis à integração universidadeserviço-comunidade.

\section{DESCRITORES}

Saúde da Família. Atenção Primária à Saúde. Aprendizagem. $\mathbf{0}$ Programa de Educação pelo Trabalho para a Saúde - PET-Saúde, é um instrumento que viabiliza programas de especialização e aperfeiçoamento em serviço dos profissionais da saúde, assim como de iniciação ao trabalho, estágios e vivências, dirigidos aos estudantes dessa área, de acordo com as necessidades do Sistema Único de Saúde (SUS). Ele deve servir como estímulo para a formação de profissionais e docentes com elevada qualificação técnica, científica, tecnológica e acadêmica, bem como uma atuação profissional pautada pelo espírito crítico, pela cidadania e pela função social da educação superior, orientados pelo princípio da indissociabilidade entre ensino, pesquisa e extensão (BRASIL, Port. No 1802, 2008).

As diretrizes curriculares dos cursos da área da saúde recomendam a inserção precoce e responsável dos acadêmicos nos serviços de saúde, permitindo assim que profissionais da atenção básica orientem e supervisionem estudantes de graduação, tendo o serviço público de saúde como cenário de ações de aprendizagem vivenciadas na prática.

O compromisso social das instituições de ensino superior contribui para que ações voltadas ao tripé ensino-pesquisa-extensão sejam construídas em ação intersetorial, envolvendo profissionais e acadêmicos em propostas que fortaleçam a formação e ações no serviço com vistas à promoção da saúde. A inserção precoce de universitários em diferentes espaços sociais é necessária à formação integral e prepara-os para a atuação profissional.

Para a execução do PET-Saúde, em 2009, na Universidade Federal de Mato Grosso do Sul (UFMS), em Campo Grande, foi firmado Convênio com a Secretaria Municipal de Saúde (SESAU), para delimitar o cenário de atuação dos acadêmicos nas Unidades Básicas de Saúde da Família, dar anuência na escolha 
dos preceptores, apontar necessidades que pudessem ser inseridas na rotina dos petianos junto às equipes das unidades escolhidas como sede das ações.

Esse estudo tem por objetivo relatar a percepção de acadêmicos do curso de Odontologia da UFMS sobre o PET-Saúde 2009 e seu impacto na formação acadêmica.

\section{MATERIAL E MÉTODOS}

Trata-se de um relato de experiência tendo como fontes de dados relatórios mensais de acadêmicos de Odontologia do PET-Saúde 2009 no Convênio UFMS/SESAU, e documentos de acompanhamento dos mesmos. Nesse estudo, os acadêmicos participantes do PET-Saúde 2009 UFMS/SESAU são denominados petianos.

\section{RESULTADOS}

\section{Um pouco de histórico}

Em outubro de 2008, tiveram início as atividades vinculadas ao PET-Saúde UFMS/SESAU, com a conformação de um grupo de docentes de cursos da área de saúde interessados em participar que se autodenominou Grupo de Condução do PET-Saúde. Esses docentes se voluntariaram a estudar a Portaria $\mathrm{N}^{\circ} 1.802 / 2008$ - MS /MEC e deram os primeiros passos na divulgação, no processo seletivo dos alunos, na escolha dos preceptores, na definição de tutores e temas de pesquisas a serem desenvolvidos. Duas docentes do Grupo manifestaram interesse pela tutoria e houve anuência. O Programa começou a ser estruturado e as minutas dos Projetos começaram a ser desenhadas pelas tutoras de cada linha de pesquisa, sempre com a colaboração de professores do Grupo de Condução e por servidores da Estratégia Saúde da Família da SESAU, para que as ações atendessem necessidades dos serviços e dos usuários das áreas que viriam a participar dos Projetos.

O processo de seleção dos acadêmicos foi sendo estruturado (currículo e entrevista), paralelamente, bem como a busca de um espaço e de infra-estrutura física que acolhesse o PET-Saúde UFMS/ SESAU 2009, o qual foi instalado na Faculdade de Medicina como estratégia para sensibilização do curso de medicina. Os processos burocráticos ficaram alocados na Pró-Reitoria de Ensino de Graduação (PREG).

Muitas reuniões se seguiram para um melhor entendimento do Programa com os docentes do Grupo de Condução e com a PREG. Seguiu-se a construção do Núcleo de Excelência, da Comissão Gestora e seus respectivos Regimentos.

\section{Apresentando os preceptores e os acadêmicos}

De acordo com a orientação da SESAU, os grupos PET-Saúde foram alocados no Distrito Sul, em seis unidades de saúde da Família. Por negociações com a SESAU, é no Distrito Sul que a UFMS concentra a maior parte das atividades práticas vinculadas aos diversos cursos da saúde.

As linhas de pesquisas escolhidas foram:

1. Educação em Saúde e Controle Social;

2. Avaliação do Projeto Viver Legal.

As tutoras, respectivamente, bióloga, doutora em saúde pública e, enfermeira e doutora em saúde pública.

A composição do grupo de preceptores para a primeira linha de pesquisa contava com uma assistente social, três enfermeiras, um médico e uma odontóloga. Na segunda linha de pesquisa havia dois odontólogos e quatro enfermeiros.

O grupo PET-Saúde foi composto por 60 acadêmicos e foram organizados nos grupos por ordem de classificação e com a preocupação em se conformar grupos multidisciplinares, levando-se em conta, ainda, a disponibilidade de seus horários. Os acadêmicos que cursavam Odontologia eram em número de 5 , representando $8,3 \%$ do número total de participantes, e deles, três eram bolsistas. Coincidentemente, todos os acadêmicos do curso de Odontologia participaram da linha de pesquisa Educação em Saúde e Controle Social. Levando-se em consideração apenas esse, os acadêmicos representaram $16,7 \%$, do total de 30 alunos.

\section{Atividades Realizadas}

Os "petianos" foram orientados, entre vários aspectos, a participar de projetos e iniciativas já existentes nos serviços de saúde, criar novas propostas de trabalho dentro das duas linhas de pesquisas e que tivessem vínculo com as prioridades das equipes de saúde da família, vivenciando uma prática interdisciplinar e multiprofissional na perspectiva de qualificar ainda mais sua formação.

No mês de maio de 2009 houve a Aula Inaugural PET-Saúde, um evento que convidou todos os cursos da área da saúde, realizada no teatro Glauce Rocha/ UFMS, para conhecerem o Programa PET-Saúde. Em agosto do mesmo ano houve o I Encontro dos grupos PET-Saúde de Mato Grosso do Sul, com a participação 


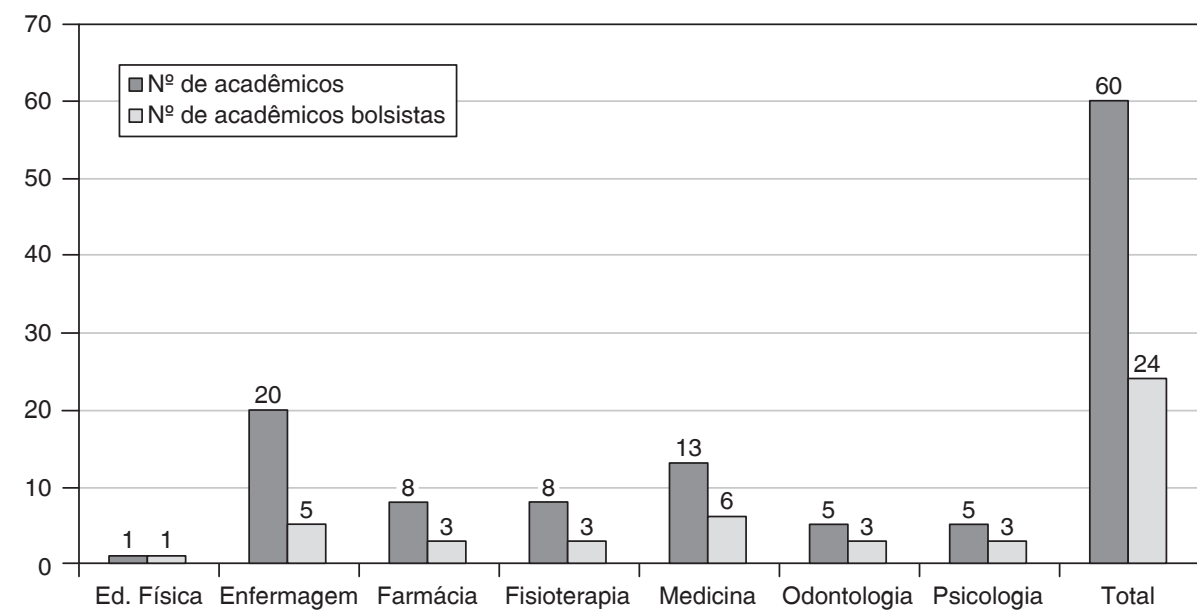

Gráfico 01 - Quantitativo de alunos por curso e alunos bolsistas PET-Saúde UFMS/SESAU, Campo Grande, 2009.

do PET-Saúde da Universidade Federal da Grande Dourados/Dourados-MS, inserido na programação da Mostra Comemorativa dos 10 anos de Saúde da Família em Campo Grande, onde os petianos puderam relatar as experiências vividas e também apresentar vários trabalhos científicos.

Como uma das primeiras atividades, foi realizada uma Oficina de sensibilização (20h), com a participação de todos os alunos e preceptores, organizada pela tutora da linha de pesquisa Educação em Saúde e Controle Social para apreensão de conceitos relativos à área de educação em saúde.

Os acadêmicos eram convocados para reuniões quinzenais em horário alternativo - das $11 \mathrm{~h}$ às $12 \mathrm{~h}$, ou das $17 \mathrm{~h}$ às $19 \mathrm{~h}$, e até aos domingos -, o que possibilitou a integração dos componentes do grupo - alunos, tutora e preceptores. Houve diversos encontros para orientação quanto à elaboração de trabalhos científicos e apresentações em Eventos. Foram realizadas, ainda, as atividades inerentes à linha de pesquisa "Educação em Saúde e Controle Social", como:

- levantamento bibliográfico,

- coleta de dados e

- participação de ações educativas dentro das Unidades de Saúde da Família parceiras do Projeto.

A rotina semanal dos petianos incluía um total de 8 horas, conforme preconizado pela Port. $\mathrm{N}^{\circ}$. 1.802/2008. Nelas estavam incluídas as atividades nas Unidades (4h). Os alunos puderam participar das reuniões do Conselho da Unidade, verificando sua dinâmica de funcionamento; reconhecimento do território da Unidade, identificação das ações educativas em saúde desenvolvidas pelos diversos componentes das equipes. Nas outras 4 horas complementares, foram feitas atividades relacionadas à pesquisa de temas relevantes para o andamento da referida linha de pesquisa, elaboração de relatórios e resumos para eventos científicos, dentre outros.

\section{A percepção dos acadêmicos de Odontologia}

Na percepção dos petianos da Odontologia ficam evidentes potencialidades e fragilidades do Programa.

\section{a) Potencialidades}

Sobre Conhecimentos adquiridos na prática

A criação do PET- Saúde foi de fundamental importância para a saúde pública, pois mantém um vínculo dos futuros profissionais da área da saúde com a Saúde coletiva, já que em ambiente da sala de aula, o que realmente existe é a teoria, deixando, na maioria das vezes, o aluno sem a real noção do impacto e da relevância que é na prática o exercício dessas ações (Acadêmico 1).

Através do PET-Saúde, tivemos a oportunidade de conhecer não só na teoria mas também na prática o funcionamento das unidades básicas de saúde da família(UBSF) bem como seus deveres com a população e os direitos da comunidade em relação aos atendimentos oferecidos (Acadêmicos 2 e 3).

\section{Sobre Multiprofissionalidade}

A integração dos cursos é um dos pontos mais importantes desse projeto, sendo o multiprofissionalismo essencial para uma verdadeira integralidade (um dos mais importantes princípios doutrinários do SUS) (Acadêmico 1).

(...) estamos certos de que multidisciplinariedade torna o trabalho muito mais produtivo (Acadêmicos 2 e 3 ). 
A proposta de interdisciplinaridade entre os acadêmicos de diversos cursos e entre os funcionários das UBSFs tem sido seguida a risca, resultando em produção de conhecimento e experiências demonstradas em cada reunião e propiciando a coleta de dados para a pesquisa do Controle Social (Acadêmico 4).

\section{Orgulho em ser petiano}

Sinto-me muito lisonjeada em poder fazer parte dessa primeira e nova geração intitulados por nós de Petianos e creio que esse é o primeiro passo de uma caminhada com muitas dificuldades, porém também de grandes e importantes conquistas (Acadêmico 1).

Sentimo-nos privilegiados por estarmos inseridos no PETSaúde, hoje somos uma grande família de amigos, unidos pela mesma causa, que vem a ser o fortalecimento do nosso sistema único de saúde contribuindo para que o mesmo, em sua universalidade, eqüidade, integralidade possa cativar seus usuários (Acadêmicos 2 e 3).

Atividades vinculadas à linha de pesquisa

Outro aspecto relevante é o maior conhecimento sobre o Controle Social e o verdadeiro papel do Conselho Gestor na unidade, em seu poder de tornar o funcionamento da mesma melhor em todos os sentidos, tanto organizacional, quanto em respeito às doutrinas do SUS. Observamos também a alienação de grande parte dos usuários sobre o papel deste conselho e principalmente na ínfima participação nas reuniões. Sendo este um projeto árduo que o grupo PET-Saúde deve desenvolver (esclarecer e conscientizar essa comunidade sobre o seu papel dentro da unidade) (Acadêmico 1).

\section{b) Fragilidades}

(...) a dificuldade maior em manter esse ponto (multiprofissionalismo) é a flexibilidade de horários, já que são cursos com uma grande carga horária (Acadêmico 1).

É difícil todos os envolvidos terem a mesma disponibilidade de horário para realizar as atividades devido à carga horária dos diferentes cursos de graduação, para estarem todos na mesma hora e no mesmo lugar, por exemplo. Esse talvez seja o maior obstáculo que o PET-Saúde enfrenta. Apesar disso, os acadêmicos mostram-se abertos ao diálogo e bem flexíveis para tomadas de decisões quanto aos horários de reuniões e metas para cumprir (Acadêmico 4).

\section{DIsCUSSÃo}

A inserção dos acadêmicos de Odontologia participantes do PET-Saúde UFMS 2009 nos serviços de saúde, atuando de forma organizada e orientada por profissionais da rede, traz elementos que agrega qualificação na formação desses futuros profissionais, ao inseri-los precocemente em atividades, práticas e vivências vinculadas às políticas de saúde pública e está de acordo com os objetivos estabelecidos na Portaria Interministerial $\mathrm{N}^{\circ} 1.802 / 2008$.

O PET-Saúde estabelece meios para que os acadêmicos desse curso concluam sua graduação preenchendo uma série de requisitos, tais como formação humanista, integral, crítica e reflexiva, para atuar em diferentes níveis de atenção à saúde, com base no rigor técnico e científico, qualificando ainda mais a sua formação. O PET-Saúde ajudou a compreender aspectos das realidades social, cultural e econômica das áreas onde eles estavam inseridos. Os acadêmicos também foram capazes de desenvolver ações de prevenção e promoção de saúde, em nível coletivo de forma integrada com acadêmicos de outros cursos, com benefícios mútuos.

O PET-Saúde, ainda, possibilitou o desenvolvimento de aspectos de liderança, comunicação, trabalho em equipe multiprofissional ligada à Estratégia de Saúde da Família (ESF), de forma interdisciplinar, possibilitando encontros e aprendizagem, evidenciando dificuldades e favorecendo a descoberta de formas de atuação com foco na promoção da saúde. Foi possível identificar nos acadêmicos participantes uma maior iniciativa, a criatividade e cidadania. Compartilhar saberes e interagir de maneira dinâmica, entendendo que todos têm papel fundamental na manutenção da saúde e defesa da vida foi, sem dúvida, um elemento motivador.

Buscando uma aproximação com as Diretrizes Curriculares Nacionais (DCNs) do curso de Odontologia o PET-Saúde permitiu, ainda, o estímulo de práticas de estudo independente, visando uma progressiva autonomia intelectual e profissional levar os alunos dos cursos de graduação em saúde a aprender a aprender que engloba aprender a ser, aprender a fazer, aprender a viver juntos e aprender a conhecer, permitindo a construção de perfil acadêmico com conteúdos, competências e habilidades através de vivências na integração ensino-serviço, no conceito ampliado de saúde, nos princípios e diretrizes do Sistema Único de Saúde (SUS), dentre eles, o atendimento integral, com prioridade para as atividades preventivas, sem 
prejuízo dos serviços assistenciais e participação da comunidade.

Conhecer e utilizar métodos e técnicas de investigação e a elaboração de trabalhos acadêmicos e científicos contribuiu para planejar e administrar serviços de saúde comunitária através de atividades extra-unidades inseridos nas equipes da ESF com vistas à construção da cidadania através de atitudes e valores correspondentes à ética profissional e ao compromisso com a sociedade.

\section{CONCLUSÕES}

Considerando as falas dos petianos constata-se um impacto relevante na formação acadêmica, pois tendo como cenário do PET-Saúde a Atenção Básica e como referenciais as DCNs e a portaria $\mathrm{N}^{\circ} 1.802$, os aspectos multiprofissionalidade, integração teoria e prática, produção de conhecimentos na área da Saúde Coletiva, princípios do SUS, funcionamento de uma UBSF, capacidade de diálogo, respeito aos colegas e tomada de decisões, ficaram evidentes.

Ficou evidenciado, também, a apreensão de aspectos do Controle Social e sua importância para a gestão da Unidade, objeto da primeira linha de pesquisa apresentada.

Acredita-se que a satisfação em ser petiano tenha sido um elemento motivador e de sensibilização para a atuação e o aprendizado decorrente da inserção precoce em UBSFs no processo de formação acadêmica. Pode-se inferir que essa satisfação seja o fato de pertencer a um grupo seleto de acadêmicos dispostos aos desafios e possibilidades que o novo possa trazer.

Espera-se que o diferencial na aprendizagem e na formação desses acadêmicos sensibilize seus pares na busca de novos conhecimentos e de Projetos que os qualifiquem para uma atuação profissional competente e cidadã. Que esse diferencial estimule os cursos a incorporarem em disciplinas e/ou módulos de maneira mais precoce a inserção dos acadêmicos em atividades práticas e vivências na Atenção Básica. Espera-se ainda que as Instituições de Ensino Superior atentem para uma maior institucionalização de Projetos com esse perfil, e que as Secretarias de Saúde dos Municípios sejam bastante permeáveis à integração universidade-serviço-comunidade, pois as atividades extra-muros têm o potencial de preparar o profissional que o Sistema de Saúde e a sociedade precisam.

\section{ABSTRACT}

Dentistry students' perceptions of the PET-Health UFMS/SESAU Program, Campo Grande, MS, 2009

Introduction: The PET-Health program should encourage the training of professionals and professors that have high technical, scientific, technological and academic qualification, as well as professional performance driven by critical thinking, citizenship and the social role of higher education, guided by the principle of indivisibility of teaching, research and extension. There was an agreement between the Federal University of Mato Grosso do Sul (UFMS) and the Municipal Health Department (SESAU) that led to the creation of a seven-course program in 2009, in two lines of research. Objectives: To report the perception of Dentistry students from UFMS concerning the PET-Health 2009 and its impact on academic education. Material and Methods: An account of an experience, supported by monthly reports by Dentistry students about the PET-Health 2009 and accompanying documents of these students as the data sources. Results: There were 60 students from seven courses in the area of Health; five were attending Dentistry courses, and three were scholarship students. There was a significant impact on academic training, insofar as aspects of multiprofessional integration of theory and practice, health-based knowledge production, SUS principles, operation of a UBSF, dialogue skills, respect for colleagues and decision-making were all put into evidence. It is believed that the satisfaction of participating in the a PET program has motivated and sensitized students for work and learning. Conclusions: It is hoped that institutions of higher education and municipal health departments will be open to the integration of university-community-service.

\section{DESCRIPTORS}

Family Health. Primary Health Care. Learning. .

\section{REFERẾNCIAS BIBLIOGRÁFICAS}

1. Brasil. Parecer CNE/CES N 1.300 , de 07 de Dezembro de 2001. Institui Diretrizez Curriculares Nacionais para o Curso de Odontologia. Diário Oficial da União, Brasília, DF, 2008.

2. Brasil. Portaria Interministerial $\mathrm{N}^{\circ} 1802$, de 26 de Agosto de 2008. Institui o Programa de Educação pelo Trabalho para a Saúde-PET - Saúde. Diário Oficial da União, Brasília, DF, 2008.

Recebido em 07/07/2011

Aceito em 25/07/2011 